\title{
Central Cell in Flowering Plants: Specification, Signaling, and Evolution
}

\author{
Hong-Ju Li** and Wei-Cai Yang ${ }^{1,2 *}$ \\ ' State Key Laboratory of Molecular Developmental Biology, Institute of Genetics and Developmental Biology, Chinese \\ Academy of Sciences, Beijing, China, ${ }^{2}$ College of Advanced Agricultural Sciences, University of Chinese Academy \\ of Sciences, Beijing, China
}

\section{OPEN ACCESS}

Edited by:

Daisuke Maruyama,

Yokohama City University, Japan

Reviewed by:

Anja Schmidt,

Universität Heidelberg, Germany Ayelen Mariana Distéfano,

Universidad Nacional de Mar del Plata

Mar del Plata, Argentina

*Correspondence:

Hong-Ju Li

hjil@genetics.ac.cn

Wei-Cai Yang

wcyang@genetics.ac.cn

Specialty section:

This article was submitted to Plant Development and EvoDevo,

a section of the journal

Frontiers in Plant Science

Received: 01 August 2020 Accepted: 28 September 2020

Published: 21 October 2020

Citation:

Li H-J and Yang W-C (2020) Central Cell in Flowering Plants:

Specification, Signaling,

and Evolution.

Front. Plant Sci. 11:590307.

doi: 10.3389/fp/s.2020.590307
During the reproduction of animals and lower plants, one sperm cell usually outcompetes the rivals to fertilize a single egg cell. But in flowering plants, two sperm cells fertilize the two adjacent dimorphic female gametes, the egg and central cell, respectively, to initiate the embryo and endosperm within a seed. The endosperm nourishes the embryo development and is also the major source of nutrition in cereals for humankind. Central cell as one of the key innovations of flowering plants is the biggest cell in the multicellular haploid female gametophyte (embryo sac). The embryo sac differentiates from the meiotic products through successive events of nuclear divisions, cellularization, and cell specification. Nowadays, accumulating lines of evidence are raveling multiple roles of the central cell rather than only the endosperm precursor. In this review, we summarize the current understanding on its cell fate specification, intercellular communication, and evolution. We also highlight some key unsolved questions for the further studies in this field.

Keywords: central cell, double fertilization, flowering plants, gymnosperm, cell specification, cell-cell communication

\section{INTRODUCTION}

Unlike that in animals where the haploid spores generated by meiosis directly differentiate into functional gametes, in plants the haploid spores undergo additional mitosis to produce multicellular gametophytes. In lower plants, like the Bryophyte, gametophytes are dominant of their life cycles and unusually free-living, whereas in seed plants (gymnosperms and angiosperms), the gametophytes are structurally reduced and develop within the sporophytic sexual organs. In flowering plants (angiosperms), the female gametophytes are developmentally reduced to a miniature structure with only a few cells embedded within layers of sporophytic ovular tissues. In contrast to the gametophyte-dominant species and sporophyte-dominant gymnosperms, the emergence of an additional female gamete, the central cell, is a critical innovation of sexual reproduction and a hallmark of angiosperms. Fertilization of both the egg and the central cell, known as double fertilization, produces the embryo and endosperm, respectively, within the seed coat. Besides acting as the endosperm precursor, the central cell also undertakes important roles during embryo sac development and function. This review outlines recent advances in our understanding of the central cell with focuses on cell specification, cell-to-cell communication, and evolution. 


\section{TYPES OF EMBRYO SAC}

In flowering plants like Arabidopsis, a single megaspore mother cell (MMC) was initiated at the tip of each nucellus (Yadegari and Drews, 2004; Shi and Yang, 2011; Yang et al., 2010; Lora et al., 2019). Through meiosis, the MMC produces four haploid megaspores, of which only one becomes the functional megaspore, the other three are degenerated (Figure 1A). The functional megaspore undergoes three rounds of nuclear mitosis to form a syncytial female gametophyte with eight nuclei. Subsequently, the syncytial female gametophyte undergoes simultaneous cytokinesis to form a typical eight-nucleated, seven-celled embryo sac (Figure 1B; Christensen et al., 1997; Shi and Yang, 2011; Yang et al., 2010). Finally, cell fate specification and maturation take place to generate the four cell types within the functional female gametophyte: two synergid cells and an egg cell at the micropyle end, a diploid central cell and three antipodal cells at the chalazal end that connects tightly with the maternal tissues (Figure 1B). This developmental pattern is known as monosporic Polygonum-type that exists in most angiosperms. In the model plant Arabidopsis, the three antipodal cells are short-lived, while in monocot, they proliferate and participate in the endosperm development. As an exception, the basal angiosperm Amborella trichopoda, a single extant species, forms a unique Amborella-type embryo sac that contains three synergid cells due to an extra cell division of one of the micropylar cells (Figure 1B; Friedman, 2006; Friedman and Ryerson, 2009). Another group of basal flowering plants, Nymphaeales (including Hydatellaceae) and Austrobaileyales, exhibits the Nuphar/Schisandra-type embryo sac that is four-celled with a haploid central cell and without antipodals (Figure 1B; Baroux et al., 2002; Friedman, 2008; Povilus et al., 2015; Zini et al., 2016). Other types of embryo sacs also exist in a number of angiosperm taxa in nature, such as the bisporic and tetrasporic types (Friedman et al., 2008; Schmid et al., 2015). All the different patterns appear to be modular with the micropylar egg-apparatus module and the chalazal module across species (Friedman and Williams, 2004). It is unknown whether the variation of female gametophyte structure among plant taxa has any adaptive significance. It has been suggested that developmental lability at the earliest stage of angiosperm evolution may lead to this variation (Friedman, 2006). Given the diverse structure, the molecular determination of the central cell may vary from taxa to taxa. This makes the generalization of the regulation of embryo sac development and its evolutionary origin difficult. Nevertheless, ubiquity of the four-cell types indicates that a few conserved factors might be enough to orchestrate the structural organization and cell fate determination. So far, our understanding on molecular regulation of female gametophyte development is mostly from the model plant Arabidopsis and a few crop species.

During the female gametophyte development, positional cues, hormones, and coordinated cell-to-cell communication have been proposed to orchestrate the establishment of embryo sac polarity and development (Kägi and Groß-Hardt, 2007; Pagnussat et al., 2007, 2009; Moll et al., 2008; Sundaresan and
Alandete-Saez, 2010; Kirioukhova et al., 2011; Lituiev et al., 2013; Martin et al., 2013; Kong et al., 2015; Panoli et al., 2015; Tekleyohans et al., 2017). A plethora of genetic factors have been identified to be involved in the development of embryo sac and its cellular differentiation (Yang et al., 2010; Chevalier et al., 2011; Erbasol Serbes et al., 2019). In the following paragraphs, specific genetic regulations of the central cell are summarized (Table 1).

\section{CENTRAL CELL DEVELOPMENT AND SPECIFICATION}

In contrast to other gametophytic cells, the central cell is characterized by its central position and large volume, including a large central vacuole at the chalazal end, two polar nuclei, cytoplasm and organelles that are inherited from the developing syncytial gametophyte. The polar nuclei fuse before fertilization in Arabidopsis, but after fertilization in cereals (Mol et al., 1994). Hence, the central cell is homodiploid (2n) in most angiosperms and polyploidy in some other species due to fusion of more than two nuclei, but remains haploid in some basal angiosperms such as Nymphaeales and Austrobaileyales (Figure 1B). The variation of the ploidy of the central cell can impact the paternal-tomaternal genome ratio that contributes to the seed size. Although central cell is the hallmark of angiosperms and prerequisite for double fertilization, molecular mechanisms controlling its development and specification are poorly understood (Berger, 2003; Yang et al., 2010).

\section{Formation of Central Cell}

Functional dissection of several genetic factors has shed light on the molecular mechanism of central cell formation. After cellularization, the two polar nuclei fuse to give rise to a large central cell nucleus, and defect of nuclei fusion would affect the function and specification of central cell. Mitochondria play an active role in polar nuclei fusion. GAMETOPHYTIC FACTOR2 (GFA2) encodes a mitochondrial chaperone that is required for the outer membrane fusion of polar nuclei (Christensen et al., 2002). Loss of function of GAMETE CELL DEFECTED1 (GCD1), a conserved mitochondrial protein, causes unfused polar nuclei, and the egg cell is also smaller compared to the wild-type, indicating that GCD1 is required for the development of both female gametes ( $\mathrm{Wu}$ et al., 2012). Mutant in mitochondrial cysteinyl t-RNA synthetase SYCO ARATH (SYCO) and ATP/ADP translocator 2 (AAC2) results in unfused polar nuclei and persistent antipodal cells (Kägi et al., 2010). In addition, endoplasmic reticulum (ER) chaperones are also involved in this process. Loss of function of Arabidopsis ER chaperone genes BiP1 and BiP2 also causes unfused polar nuclei (Maruyama et al., 2010). $\mathrm{BiP}$ proteins can interact with ER-resident J-domain protein to mediate polar nuclei membrane fusion (Maruyama et al., 2014). The J-domain proteins ERdj3A and P58IPK mediate outer nuclear membrane fusion, while ERdj3B and P58IPK regulate inner nuclear membrane fusion. RNA metabolism 
A

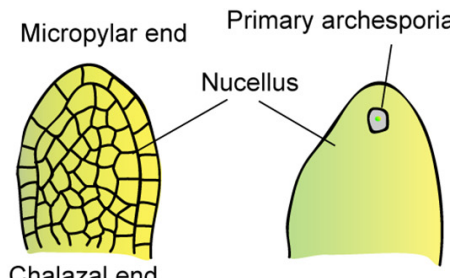

Megaspore mother cell (MMC)

Chalazal end

B

Polygonum type
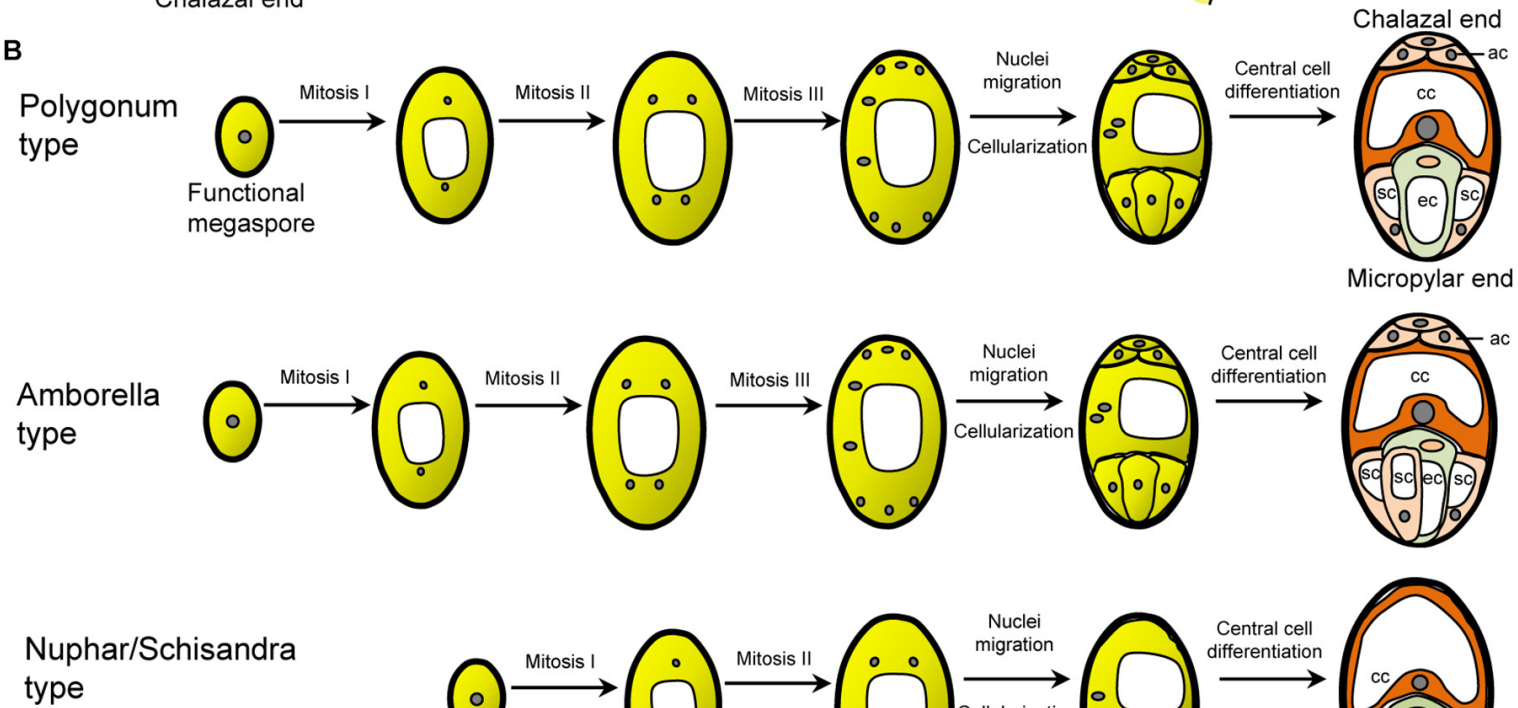

C
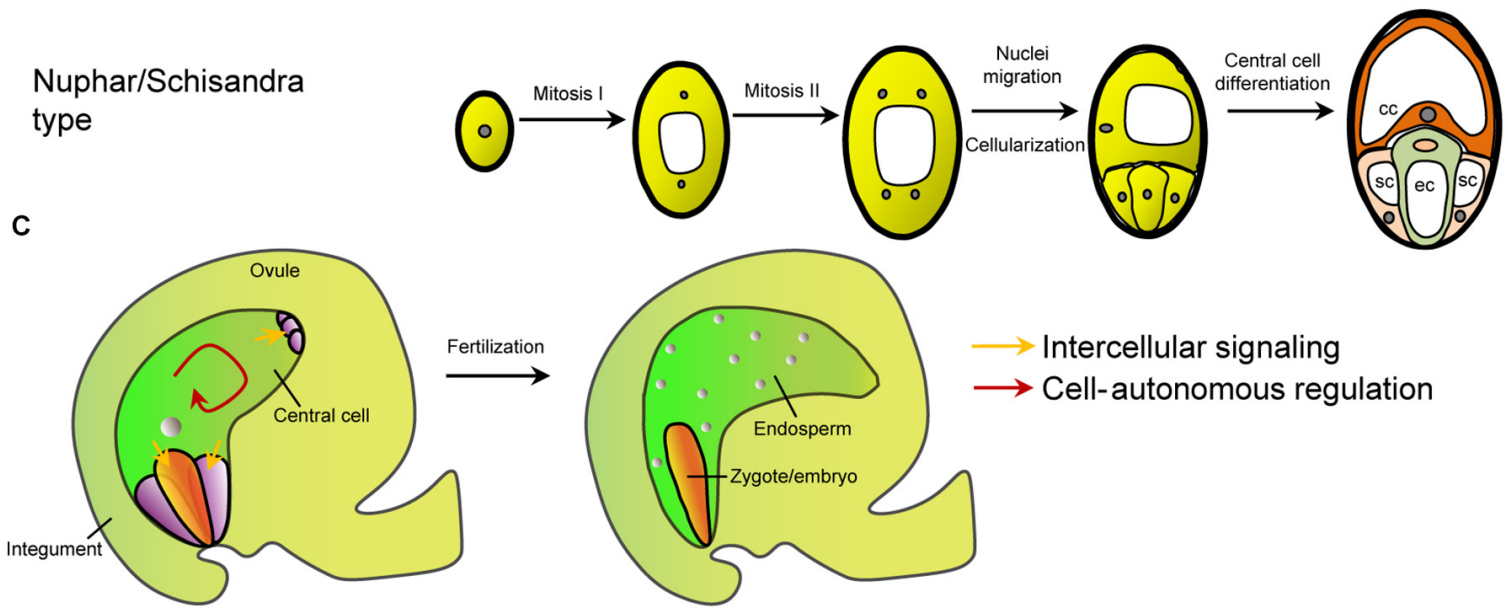

FIGURE 1 | The female gametophyte patterns and intercellular signaling between central cell and other cells. (A) Schematic diagram of the megasporogensis. (B) Schematic diagram of three female gametophyte types mentioned in the text. The Polygonum type is exhibited by most (>70\%) flowering plants, including Brassicaceae, Gramineae, Malvaceae, Leguminoseae, and Solanaceae (Yadegari and Drews, 2004). The Amborella and Nuphar/Schisandra types are the primitive ones. Other types can be referred to reviews by Yadegari and Drews (2004), Schmid et al. (2015), and Gonzalez et al. (2019). White shapes within the cells represent vacuoles. (C) Schematic diagram of the female gametophyte within an Arabidopsis ovule and embryo and endosperm after fertilization. Arrows, the intercellular and cell-autonomous signaling.

and processing are also involved in central cell development. A homolog of yeast RNA helicase MAA3 (MAGATAMA3) is required for polar nuclei fusion and pollen tube attraction (Shimizu et al., 2008). Arabidopsis genes LACHESIS (LIS), CLOTHO (CLO/GFA1), and ATROPOS (ATO) encode core spliceosomal components and are initially expressed throughout the female gametophyte. Defect of these genes causes the switch of the synergids and central cells to the fate of egg cells (Groß-Hardt et al., 2007; Moll et al., 2008). In lis and clo mutant, polar nuclei also fail to fuse (GroßHardt et al., 2007; Völz et al., 2012). Furthermore, Soluble N-Ethylmaleimide-Sensitive Fusion Protein Attachment Protein
Receptors (SNARE) gene SEC22 is also involved in polar nuclei fusion (El-Kasmi et al., 2011). ROS accumulation is correlated with the activation of central cell reporter genes, thus ROS may also be involved in central cell development (Martin et al., 2013). Moreover, wyrd (wyr) mutant of the putative plant ortholog of the Inner Centromere Protein (INCENP), produces additional egg cells at the expense of the accessory synergid cells and is defective in central cell differentiation (Kirioukhova et al., 2011). These studies highlighted the roles of mitochondria, ER, membrane dynamics, and RNA processing in the maturation of the central cell (Table 1). 
TABLE 1 | Summary of genes involved in central cell function and specification.

\begin{tabular}{|c|c|c|c|}
\hline Gene name & Gene description & Function in FG/Phenotype & Molecular machinery/Cellular process \\
\hline AGL80 & MADS box protein & Central cell switch to accessory cells & Transcription regulation \\
\hline AGL61 & MADS box protein & Central cell switch to accessory cells, central cell degeneration & \\
\hline TPL & Transcription co-repressor & Central cell specification & \\
\hline CCG & TFIIB family & Central cell-mediated pollen tube attraction/Failed pollen tube attraction & \\
\hline CBP1 & Components of transcription complex & & \\
\hline GFA2 & Homolog of yeast Mdj1p, chaperone & Failed synergid cell death and polar nuclei fusion & Mitochondria function \\
\hline AAC2 & ATP/ADP translocator & Unfused polar nuclei, persistent antipodal cells and reduced egg cell size & \\
\hline SYCO/FIONA & Cysteinyl t-RNA synthetase & Life span of the antipodal cells, failed polar nuclei fusion & \\
\hline GCD1 & A conserved mitochondrial protein & Unfused polar nuclei, smaller egg cell & \\
\hline CLO & Spliceosomal components & Unfused polar nuclei, switch of the synergids and central cells to the fate of egg cells & RNA processing \\
\hline \multicolumn{4}{|c|}{ 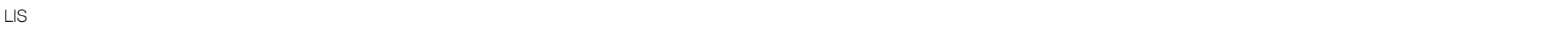 } \\
\hline \multicolumn{4}{|l|}{ ATO } \\
\hline MAA3 & RNA helicase & Unfused polar nuclei, pollen tube attraction & \\
\hline Bip1/2 & ER chaperones & Unfused polar nuclei & ER homeostasis \\
\hline \multicolumn{4}{|l|}{ ERdj3A/B } \\
\hline \multicolumn{4}{|l|}{ P58IPK } \\
\hline SEC22 & SNARE protein & Unfused polar nuclei & Membrane dynamics \\
\hline WYR & Ortholog of the Inner Centromere Protein (INCENP) & Central cell differentiation, additional egg cells & Chromosome regulation \\
\hline DME & DNA glycosylase & Required for maternal expression of imprinted genes in the central cell & \\
\hline FIS2, MEA, FIE & PRC2 complex & Inhibit cell autonomous endosperm development & \\
\hline CKI1 & Histidine kinase & Cell fate of central cell and antipodal cells failed polar nuclei fusion & Cytokinin signaling pathway \\
\hline
\end{tabular}




\section{CKI1 Signaling Pathway}

Genetic studies demonstrated that cytokinin signaling pathway is required for female gametophyte development and specification of central cell fate (Pischke et al., 2002; Hejátko et al., 2003; Deng et al., 2010; Cheng et al., 2013; Yuan et al., 2016). Disruption of the two-component sensor histidine kinase CKI1, an activator of the cytokinin signaling pathway, causes abortion of the central vacuole and degradation of the female gametophyte after completion of three mitotic divisions (Pischke et al., 2002; Hejátko et al., 2003; Deng et al., 2010). Later using cell-specific fluorescent markers, it was found that the egg marker is misexpressed in all nuclei while the central cell marker is either shut down or misexpressed in antipodal nuclei, and with synergid marker expressed in the central cell in the syncytial ckil mutant embryo sac (Yuan et al., 2016). These indicate that the central cell was switched to the egg cell fate in $c k i$ mutant. Consistently, ectopic overexpression of CKI1 can switch on the central cell-specific markers in the micropylar gametophytic cells (Yuan et al., 2016). These data suggest that CKI1 is required for the specification of the central cell and antipodal cells and also restriction of the egg cell fate in the central cell. Interestingly, CKI1 protein is ERlocalized and initially spread all over at two-nucleate stage and later restricted to the chalazal portion of the syncytial embryo sac at eight-nucleate stage, and concentrated around the central cell nucleus in mature embryo sac (Yuan et al., 2016). This coincides with the enriched cytokinin signaling in the chalazal due to local cytokinin biosynthesis and receptor expression (Cheng et al., 2013). Nevertheless, it remains unknown whether cytokinin itself plays a direct role or not, since CKI1 can activate downstream cytokinin signaling independent of cytokinin and lacks cytokinin-binding ability (Kakimoto, 1996; Yamada et al., 2001). Additionally, the central cell expression of IPT8 for cytokinin biosynthesis can partially rescue ckil female gametophyte lethal phenotype, suggesting that the activation of cytokinin receptor signaling can to some extent complement the loss of CKI1 (Deng et al., 2010). Of note, the ckil mutant also shows failed polar nuclei fusion (Yuan et al., 2016; Zhang et al., 2020).

The dynamic localization of CKI1 protein also implies a role of polar nuclei movement for central cell specification. How CKI1 specifies central cell fate and the role of cytokinin remain to be investigated (Weijers, 2016). There is evidence that CKI1 acts upstream of histidine phosphotransfer proteins (AHPs), which are required for female gametophyte development as well (Deng et al., 2010; Cheng et al., 2013). AHPs are also involved in central cell and antipodal cell fate determination (Liu et al., 2017). And mutation of MYB116, a proposed target of CKI1 signaling, also affects female gametophyte development (Rabiger and Drews, 2013). Together, these data strongly suggest that CKI1-mediated signaling pathway plays a critical role in central cell specification.

\section{Transcriptional Control of Central Cell Fate}

Previous studies have identified pairs of MADS-box transcription factors of the AGL family that are specifically expressed in the central cell (Bemer et al., 2010). These transcription factors often act as hetero- or homodimer to bind CArG box of target genes. Both AGL80 and AGL61/DIA are expressed specifically in the central cell and can form a heterodimer. Loss of either AGL80 or AGL61/DIA function impairs central cell maturation and renders central cell non-functional (Portereiko et al., 2006; Bemer et al., 2008; Steffen et al., 2008). Recently, it was reported that the central cell of agl80 mutant ectopically expresses synergid- and antipodal-specific marker genes (Zhang et al., 2020). This indicates that AGL80-AGL61/DIA complex is required for specification of central cell fate. Except for the type I MADS-box DNA binding domain, AGL80, but AGL61/DIA, contains a transcription repression domain, the EAR motif that is essential for AGL80 function and required for its interaction with the co-repressor TOPLESS (TPL) proteins (Zhang et al., 2020), suggesting that AGL80 acts as a transcription repressor in the central cell. Recent data, indeed, showed that AGL80 represses transcription of the synergidsspecific MYB98 genes, the major determinant factor of the synergid cell fate, in the central cell by directly binding to the CArG boxes present in the upstream promoter region of MYB98 gene (Zhang et al., 2020). Consistently, ectopic expression of AGL80 in synergids can repress the expression of MYB98, and switches on the transcriptional expression of central cell-specific gene DD22 in the synergid (Zhang et al., 2020). In addition, AGL80 can form a homodimer in Arabidopsis protoplasts. Nevertheless, it's also possible that ectopic expression of AGL80 in the synergids can also switch on the expression of AGL61. This implies that AGL80 orchestrate gene transcription, and the EAR motif-mediated transcriptional repression plays a critical role in restricting central cell fate. It remains to be determined whether other AGLs in the central cell are also required for central cell function and the roles of the AGL80 orthologs in cereals. AGL80 is required for the expression of DEMETER (DME), a DNA glycosylase that is required for DNA demethylation in the central cell, as well as the determination of central cell and endosperm development (Portereiko et al., 2006; Park et al., 2016). These findings suggest that AGL80 plays a critical role to orchestrate the epigenetic pathway in the central cell.

\section{Epigenetic Control of Central Cell}

DME-mediated demethylation is required to activate the transcription of the Polycomb Repressive Complex 2 (PRC2) components in the central cell (Köhler et al., 2012). Disruption of the PRC2 complex causes simultaneous nuclei division of the central cell before fertilization and seed abortion (Köhler et al., 2012; Raissig et al., 2013; Schmidt et al., 2013). These suggest active involvement of epigenetic regulation in the maintenance of the central cell.

At epigenetic regulation level, the central cell is drastically distinct from the egg cell. The EAR motif-containing repressors suppress target gene expression through chromatin modification of regulatory regions by histone deacetylation, often via forming complex with co-repressor TPL and histone deacetylases. In central cell of Arabidopsis and rice, locus-specific and 
active DNA demethylation contribute to the maternal chromosome hypomethylation in the endosperm (Park et al., 2016). The repressive mark $\mathrm{H} 3 \mathrm{~K} 9 \mathrm{me} 2$ was found to distinctly distribute in the egg cell, central cell and their fertilization products, suggesting epigenetic dimorphism between the two female gametes (Pillot et al., 2010). The euchromatin distributed protein LHP1/TFL2 is associated with silenced loci enriched in $\mathrm{H} 3 \mathrm{~K} 27 \mathrm{me} 3$ and shows lower expression in the central cell than the egg cell (Pillot et al., 2010). It's interesting that this dimorphism is established after embryo sac cellularization (Pillot et al., 2010). The epigenetic dimorphism is also suggested by the expression of different histone isoforms in the two female gametes (Ingouff et al., 2010). Furthermore, central cell undergoes demethylation at small nucleosome-depleted transposable elements (Ibarra et al., 2012). In addition, central cell is evidenced to be transcriptionally more active than the egg cell as shown by the immunostaining of active RNA polymerase II (Garcia-Aguilar et al., 2010). These studies suggest more active chromatin state in the central cell than the egg cell which is apparently correlated with the transcriptional activity. This epigenetic dimorphism has been proposed to be biologically significant but still waits further evidence (Baroux et al., 2011). Detailed discussion on the epigenetic regulations in the female gametophyte, including the difference between the central cell and other sister cells can be referred to other reviews (Armenta-Medina et al., 2011; Köhler et al., 2012; Kawashima and Berger, 2014; Ashapkin et al., 2019).

\section{RNA Processing in Central Cell Specification}

Except for transcriptional regulation, RNA processing pathway also participates in central cell specification as discussed above. In lis, clo, and ato mutants, the central cell and egg cell identities are misspecified (Groß-Hardt et al., 2007; Moll et al., 2008; Völz et al., 2012). In clo/+ mutant, the mutant antipodal cells adopts central cell fate as exhibited by changed position, membrane disintegration, nuclei fusion, and transcriptional activation of central cell marker genes (Moll et al., 2008).

In summary, these studies suggest multiple-layered and complex regulation of central cell fate and full understanding of the underlying mechanism is still a long way to go.

\section{CELL-CELL COMMUNICATION BETWEEN CENTRAL CELL AND ITS NEIGHBORS}

Cell-cell communication is ubiquitous in plant development and stress response. In the embryo sac, the intercellular signaling has been suggested to be critical for its development and function. The central cell directly contacts with all the sister cells in the embryo sac, which supports its extensive intercellular interaction (Figure 1C).

\section{Central Cell Control on the Egg}

The ubiquitously expressed mitochondria-localized protein GCD1 is required for the mutual signaling between the egg and central cell (Wu et al., 2012). Expression of GCD1 in either the egg cell or the central cell can rescue the embryo sac defect. It is reasonable to speculate that this cell-cell communication is important for the developmental coordination of the two dimorphic female gametes. The GCD1 is a conserved protein, but its molecular function in the central cell is currently unclear (Wu et al., 2012).

\section{Central Cell Control on Synergid}

Laser ablation experiment on the central cell suggests noncell autonomous regulation of the central cell in the full differentiation of the synergid (Susaki et al., 2015). This regulation was also evidenced by the genetic regulation of the central cell-expressed $C C G$ and $C B P 1$ on the transcription of the synergid-expressed $M Y B 98$ gene and pollen tube attractants (Chen et al., 2007; Li et al., 2015; Meng et al., 2019). CCG is an early transcribed gene in the central cell (Zhang et al., 2020). The expression of CCG in the central cell is not affected in agl80 mutant embryo sac that fails to specify the central cell fate (Zhang et al., 2020). Recently, MYB98 was shown to be expressed before cellularization, which raises the possibility that the cell-cell communication might be traced back to the nucleus-nucleus communication in the coenocyte (Susaki et al., 2020). It's still unclear whether CCG transcription is also initiated before cellularization. The central cell determinant factor AGL80GFP fusion protein was initially detected right before the polar nuclei fusion and MYB98 expression in the synergids is not affected in agl80 mutant (Portereiko et al., 2006; Zhang et al., 2020). These findings imply that the central cell-synergid intercellular communication likely initiates early during the differentiation of these cells.

\section{Central Cell Control on Antipodal Cells}

The central cell-expressed mitochondria-localized cysteinyl t-RNA synthetase FIONA/SYCO regulates the life span of the antipodals in a non-cell autonomous manner (Kägi et al., 2010). Interestingly, targeted disruption of the electron transport chain in the central cell mitochondria revealed that the lifespan of antipodal cells is coupled to the metabolic activity of the central cell (Kägi et al., 2010). This suggests that the metabolic state of the central cell is correlated with the differentiation of the surrounding cells.

\section{Molecular Mechanism of Intercellular Signaling}

The intense cell-to-cell communication between the four cell types could be quite necessary and intriguing as one considers the embryo sac is just a reduced "parasitic" miniature derived from individual plants in the long history. The underlining molecular mechanism of these intercellular signaling is unknown and several lines of evidence provide some clues. Several mechanisms, such as signaling through the extracellular matrix molecules and symplastic trafficking, are 
evidenced or proposed to be involved during female gametophyte maturation and function.

Symplastic connection is the cellular channel of intercellular transport of transcription factors, small RNAs or other small molecules and has been suggested to function in intercellular transport of small molecules within the embryo sac (Han et al., 2000; Li et al., 2015, 2018; Erdmann et al., 2017). Using fluorescent dyes, it was clearly shown that symplastic connection exists within the embryo sac. In Torenia fournieri embryo sacs, molecules less than $10 \mathrm{kDa}$ can diffuse freely between gametophytic cells, while molecules larger than $10 \mathrm{kDa}$ cannot (Han et al., 2000). More recent data showed that molecules up to $20 \mathrm{kDa}$, including 24-nucleotide small RNAs, can move between the central cells and egg cells in Arabidopsis, while this movement capacity is lost after fertilization (Erdmann et al., 2017). The small RNAs produced in the endosperm and even central cell are speculated to move to the egg and embryo to direct DNA methylation (Ibarra et al., 2012). These observations strongly suggest that material exchange and cell-cell communication exist within the embryo sac, but compelling genetic evidence and the underlining cellular support, such as the presence of plasmodesmata, for this movement still need further exploration.

Apoplastic peptides are widely employed in intercellular signaling in plants. Transcriptome profiling revealed that diverse secreted peptides are highly enriched in the embryo sac. In Arabidopsis, $c c g$ mutant ovules lose the ability to attract pollen tube (Chen et al., 2007). It has been shown that in $c c g$ ovules, more than a hundred of secreted peptide-encoding genes are down-regulated ( $\mathrm{Li}$ et al., 2015). These peptides are highly expressed in the central cells and secreted to the apoplast and even to the intercellular space of the integuments ( $\mathrm{Li}$ et al., 2015). It is possible that some protein factors required for the later endosperm development have been activated in advance in the central cell before fertilization. Three central cells-expressed cysteine-rich peptides, ESF1s, were identified to be required for the development of embryo suspensor (Costa et al., 2014). These ESF1s are also down-regulated in $c c g$ ovules (Li et al., 2015). It is still a mystery whether the numerous secreted peptides down-regulated in $c c g$ have roles in cell-cell communication between the central cell and its neighbors or intercellular signaling between the fertilization products. This type of regulation has been reported, for example, in maize the egg cell-expressed secreted peptide ZmEAL1 regulates the cell fate of the antipodal cells (Krohn et al., 2012). In addition, one clue for the role of exosome signaling is the detection of expression of tetraspanin family members in the embryo sac (Boavida et al., 2013), since tetraspanins in plant were shown to be required for exosome formation that is required for the release of anti-pathogen small RNAs (Cai et al., 2018). However, the function of these tetraspanins in embryo sac is still unknown as the mutants exhibit no phenotype.

\section{Distinction Between Central Cell and Egg Cell in Fusion With Sperms}

Double fertilization entails fusion of sperm cells with the two dimorphic female gametes, the egg and the central cell. The potentiality of sperm cells for fertilization is activated by the $E G G$ CELL1s (EC1s) that are expressed specifically in the egg cell, but not in the central cell (Márton et al., 2005). It is not known whether the central cell employs its own way to activate the sperm or takes advantage of the egg-secreted EC1. The putative receptor for gamete adhesion, GEX2, contains an extracellular domain and is required for the sperm fusion with both female gametes (Mori et al., 2014). This implies the presence of similar or the same ligand on the surface of egg and central cell. Live imaging studies showed a lagged sperm fusion with the central cell than the egg cell, indicating that differences between the two fusion events may exist (Denninger et al., 2014; Hamamura et al., 2014). Study with the polyspermic tetraspore (tes) mutant has suggested that polyspermy block exists in egg, but not in central cell (Scott et al., 2008). Other reports suggest that the egg cell can also fuse with two sperms to form triploid embryo with the central cell fuses with one sperm cell (Grossniklaus, 2017; Mao et al., 2020). Another distinction between the central cell and the egg cell is that plasma membrane fusion with sperm can trigger central cell mitotic division, suggesting signaling link between plasmogamy and activation of the nuclear division (Aw et al., 2010).

\section{EVOLUTION OF CENTRAL CELL}

The evolutionary origin of the double fertilization that is characterized by the emergence of the central cell is still mysterious, since the double fertilization phenomenon was discovered in the late 1890s. Based on the diversity of embryo sac, different hypothesis were raised to explain the evolution of different ploidy of endosperms and the adaption significance, i.e., the origin of structural novelty and its relative fitness (Friedman et al., 2008). The developmental evolution of the embryo sac is tightly related to the endosperm genetics and the variation of endosperms will gives rise to phenotype variations that are subject to natural selection. Among the extant flowering plants, seven types of endosperms exist. But our understanding on its evolutionary trajectory, adaption and even molecular modulation is still very limited.

Genetic evidence in model plant Arabidopsis has now provided new clues on the central cell evolution. AGL80 lossof-function mutant was recently found to be featured by the failed fusion with sperm cell and switch of marker genes to the accessory cells. The study of AGL80 suggests a conserved mechanism of central cell determination in Brassicaceae by the EAR-motif mediated gene repression mechanism (Zhang et al., 2020). This indicates multiple divergence of central cell specification during evolution and more primitive mechanisms are to be found. It would be interesting to investigate whether AGL80 homologs in other species are also involved in central cell specification or function even if EAR motif is not present. Although the EAR motif is only conserved in Brassicaceae, whether other transcription repression mechanism executed by AGL80 homologs or other transcription factors in monocot and other taxa are still to be unveiled. From this study, it appears that the determination of central cell fate is not conserved as expected, instead it has originated more than one time. The evolutionary 
conservation of AGL80 in the central cell in the Brassicaceae family may reflect fast evolution of the central cell that is wired for postzygotic reproductive isolation. The incompatibility of two species in the hybrid endosperm constitutes one of the major postzygotic isolation.

In gymnosperms (the non-flowering seed plants), like Cycads, Gnetales, and Gingko, the surrounding gametohpytic cells take the role of endosperm function to nourish the embryo. The female gametophyte undergoes numerous rounds of mitosis to produce a coenocytic cell with approximately thousands of free nuclei (Figure 2; Soma, 1997). At maturation, the egg-containing archegonia are structurally alike that of the moss (Figure 2), and the pollen grain contains two sperm cells at maturity and only one sperm cell would transmit the genome to the progeny. It was suggested that the gametophytic cells surrounding the archegonia function analogously to the nourishing endosperm in angiosperms. These multicellular gametophytes store reserves before fertilization to support the embryogenesis after the egg cell fertilization. With the emergence of the central cell, the embryonourishing role shifts from the gametophyte to the fertilized central cell, the endosperm. It confers the flowering plants several benefits and at the same time the fertilization process become more complex. The fertilization-dependent nutrients allocation to the endosperm saves energy as embryogenesis is not always a hundred percent successful. On the other hand, endosperm, containing the genome of both parents, takes a major part in the post-zygotic hybrid barrier (Lafon-Placette et al., 2017), to help maintain the identity of species by reducing gene flow.

The evolution of the endosperm in flowering plants has been discussed for years (Berger, 2003; Friedman et al., 2008). One scenario of the origin of the endosperm is a sexualized gametophytic cell and fertilization triggers the mitotic progression of the nourishing tissue. This scenario is supported by the similar pattern between the endosperm development of angiosperms and the female gametophyte of gymnosperms. In addition, the fertilization-dependent endosperm development gives rise to the possibility of parental regulation. Another scenario of endosperm origination is that an altruistic embryo finally takes the role of embryo nourishing. At the present time, however, there is still no evidence to show the evolutionary origin of the central cell, as no transitional or primordial female gametophyte structure has been found. Even in the most ancient flowering plant, Amborella, the four-cell-typed female gametophyte has already formed. Two recent studies suggested that the signaling component of cytokine, CKI1 in Arabidopsis regulate the central cell fate, and the orthologous CKI1 gene in Ginkgo, is expressed in archegonia and the precursor and surrounding tissues (Yuan et al., 2016, 2018). This study implies that at least some factors involved in female gametophyte development have been employed in the gymnosperm.

Although no fertilization-based endosperm was generated in gymnosperms, some comparison has been made between gymnosperms Gnetales and angiosperms (Friedman, 1998). In Gnetales, the binucleate sperm is carried by the pollen and released into the binucleate egg cell. It was reported that in Gnetales, the egg cell contains two nuclei, the centrally placed one as the egg nucleus, another one is the ventral canal nucleus (Figure 2). In Welwitschia, only the egg nucleus is fertilized since the second sperm nuclei does not enter the egg cell and degenerates (Friedman, 2015). In Gnetum and Ephedra, the two sister nuclei of the egg cell fused with the two sister sperm nuclei, respectively (Friedman, 1990). However, the coenocytic female gametophyte matures upon the pollen tube penetration and the simultaneous fertilization of the two haploid female nuclei determines the following fate of the fertilization products. But this lack of egg differentiation is unique in Gnetales and Welwitschia (Friedman, 1998). After fertilization, the conenocytic tissue undergoes cellularization and both fertilization products initiate embryogenesis and only one matures with the nourishment of the surrounding gametophytic tissues. Despite the developmental similarities of the mitotic sister sperm nuclei within the same pollen and sister female gametophytic nuclei, no definitive homology of the double fertilization events between Gnetales and flowering plants has been drawn. Another remaining mystery is the single fruitful fertilization at the expanse of waste of the other sperm. The emergence of two sperms likely have driven and provided the prerequisites for the origination of the second fertilizationcompetent cell and double fertilization. In the absence of fossil record of species with central cell ancestor, the elucidation of the evolutionary ontogeny of this specialized cell is difficult. Genetic dissection and molecular evolution study of key genes especially that with conserved roles in central cell specification would be helpful, which benefit from the release of more and more whole genome sequences of different plant taxa. High through-put transcriptome sequencing of small amount of samples would also promote the unraveling of the genetic hierarchy and evolution of female gametophyte, although the functional study would be challenging due to the difficulty in genetic transformation of these species.

\section{PERSPECTIVE}

Nowadays, we have a more comprehensive understanding of the cell specification and intercellular signaling of central cell in molecular and evolutionary aspects. The active involvement of central cell in diverse aspect of fertilization points to an emerging importance of this non-heritable female gamete. Experimental evidence is still limited for the full understanding of this mysterious cell. Although with the studies in the past two decades, the identification of the key components and their functional connectivity remains the major hurdle in understanding of central cell function and evolution. The central cell is enriched in secreted peptides, but most of them have yet to be functionally characterized as the conventional T-DNA and gene knock-down approaches are powerless in these highly redundant and sequence-diverged gene families. In addition, the relaxation of the gene silencing machinery activates the transposable elements and a large number of genes that would make the reverse genetic study laborious. With the dawn of innovative strategies in experimental techniques, such as single-cell transcriptome and epitranscriptome, gene editing 

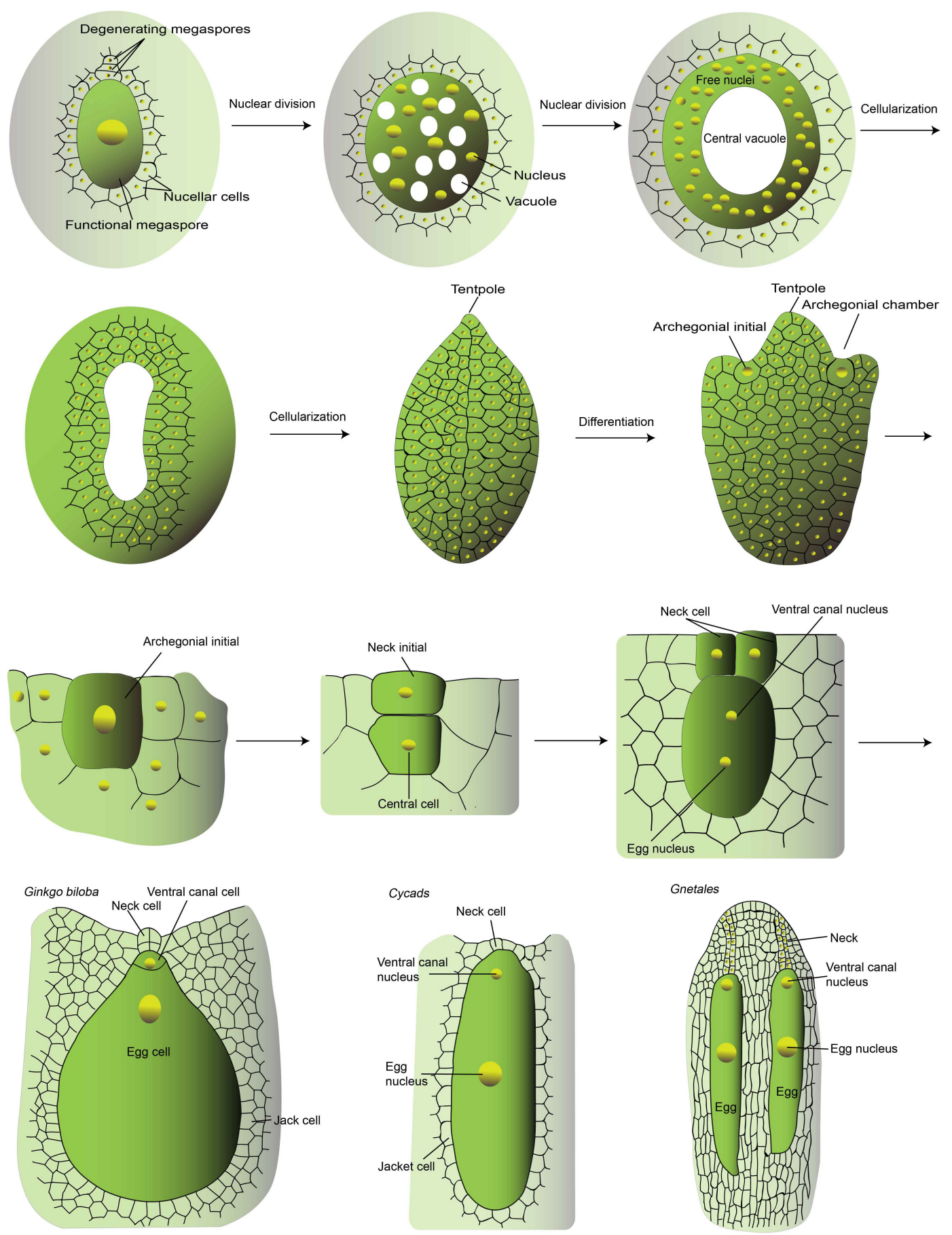

FIGURE 2 | The development of Ginkgo archegonium. This is a schematic diagram of the major stages of female gametophyte development of Ginkgo and the mature female gametophyte of Cycads and Gnetales. The schematic diagram was drawn according to Dhote, Gupta and Bijoy G (www.biologydiscussion.com) and Wang et al. (2014). The female gametogenesis of different gymnosperms is quite similar. The development of Ginkgo female gametophyte is described as a representative. In brief, it develops from the large functional megaspore, the remaining spore after meiosis. The nucleus of the megaspore divides mitotically to generate thousands of free nuclei within a cell with a large central vacuole. Thereafter, the cellularization takes place in a centripetal fashion and finally the vacuole is obliterated. The cellularized gametophyte is usually called endosperm, because it undertakes the role of embryo-nourishing like the fertilization-generated endosperm in flowering plants. Then the archegonium formation initiates. Two to four cells differentiate into the archegonial initials, which then divide periclinally to form an outer small primary neck initial and a large central cell. The primary neck initials divide vertically twice resulting in four neck cells. The central cell divide asymmetrically to generate the upper ventral canal cells which disappears quickly and a large egg cell. With the expansion of the egg cell, the neck cells are pushed outside and finally degenerate to form the opening for the sperm entry. In Cycads and Gnetales, the ventral canal nucleus and the egg nucleus are within the same cell without cell wall separation. In Gnetales, a file of neck cells are formed thus generating a longer canal for sperm entry. 
technology and increase of sequenced species, we can expect a more comprehensive understanding on central cell specification, fertilization, and coordination with the surrounding cells, as well as how the central cell helps to shape the flowering plants' overwhelming predominance on earth.

\section{AUTHOR CONTRIBUTIONS}

H-JL and W-CY wrote the manuscript. Both authors contributed to the article and approved the submitted version.

\section{REFERENCES}

Armenta-Medina, A., Demesa-Arevalo, E., and Vielle-Calzada, J. P. (2011). Epigenetic control of cell specification during female gametogenesis. Sex. Plant Reprod. 24, 137-147. doi: 10.1007/s00497-011-0166-z

Ashapkin, V. V., Kutueva, L. I., Aleksandrushkina, N. I., and Vanyushin, B. F. (2019). Epigenetic regulation of plant gametophyte development. Int. J. Mol. Sci. 20:3051. doi: 10.3390/ijms20123051

Aw, S. J., Hamamura, Y., Chen, Z., Schnittger, A., and Berger, F. (2010). Sperm entry is sufficient to trigger division of the central cell but the paternal genome is required for endosperm development in Arabidopsis. Development 137, 2683-2690. doi: 10.1242/dev.052928

Baroux, C., Raissig, M. T., and Grossniklaus, U. (2011). Epigenetic regulation and reprogramming during gamete formation in plants. Curr. Opin. Genet. Dev. 21, 124-133. doi: 10.1016/j.gde.2011.01.017

Baroux, C., Spillane, C., and Grossniklaus, U. (2002). Evolutionary origins of the endosperm in flowering plants. Genome Biol. 3:1026.

Bemer, M., Heijmans, K., Airoldi, C., Davies, B., and Angenent, G. C. (2010). An atlas of type I MADS box gene expression during female gametophyte and seed development in Arabidopsis. Plant Physiol. 154, 287-300. doi: 10.1104/pp.110. 160770

Bemer, M., Wolters-Arts, M., Grossniklaus, U., and Angenent, G. C. (2008). The MADS domain protein DIANA acts together with AGAMOUS-LIKE80 to specify the central cell in Arabidopsis ovules. Plant Cell 20, 2088-2101. doi: 10.1105/tpc.108.058958

Berger, F. (2003). Endosperm: the crossroad of seed development. Curr. Opin. Plant Biol. 6, 42-50. doi: 10.1016/s1369526602000043

Boavida, L. C., Qin, P., Broz, M., Becker, J. D., and McCormick, S. (2013). Arabidopsis tetraspanins are confined to discrete expression domains and cell types in reproductive tissues and form homo- and heterodimers when expressed in yeast. Plant Physiol. 163, 696-712. doi: 10.1104/pp.113.216598

Cai, Q., Qiao, L., Wang, M., He, B., Lin, F. M., Palmquist, J., et al. (2018). Plants send small RNAs in extracellular vesicles to fungal pathogen to silence virulence genes. Science 360, 1126-1129. doi: 10.1126/science.aar4142

Chen, Y. H., Li, H. J., Shi, D. Q., Yuan, L., Liu, J., Sreenivasan, R., et al. (2007). The central cell plays a critical role in pollen tube guidance in Arabidopsis. Plant Cell 19, 3563-3577. doi: 10.1105/tpc.107.053967

Cheng, C. Y., Mathews, D. E., Schaller, G. E., and Kieber, J. J. (2013). Cytokinindependent specification of the functional megaspore in the Arabidopsis female gametophyte. Plant J. 73, 929-940. doi: 10.1111/tpj.12084

Chevalier, E., Loubert-Hudon, A., Zimmerman, E. L., and Matton, D. P. (2011). Cell-cell communication and signalling pathways within the ovule: from its inception to fertilization. New Phytol. 192, 13-28. doi: 10.1111/j.1469-8137. 2011.03836.x

Christensen, C. A., Gorsich, S. W., Brown, R. H., Jones, L. G., Brown, J., Shaw, J. M., et al. (2002). Mitochondrial GFA2 is required for synergid cell death in Arabidopsis. Plant Cell 14, 2215-2232. doi: 10.1105/tpc.002170

Christensen, C. A., King, E. J., Jordan, J. R., and Drews, G. N. (1997). Megagametogenesis in Arabidopsis wild type and the Gf mutant. Sex. Plant Reprod. 10, 49-64. doi: 10.1007/s004970050067

Costa, L. M., Marshall, E., Tesfaye, M., Silverstein, K. A., Mori, M., Umetsu, Y., et al. (2014). Central cell-derived peptides regulate early embryo patterning in flowering plants. Science 344, 168-172. doi: 10.1126/science.1243005

\section{FUNDING}

This study was supported by the National Natural Science Foundation of China (31870295 to H-JL and 31991203 to W-CY) and the Ten Thousand Talent Program (Grant W03070036).

\section{ACKNOWLEDGMENTS}

We apologize for not able to refer to some related literatures due to page limit.

Deng, Y., Dong, H., Mu, J., Ren, B., Zheng, B., Ji, Z., et al. (2010). Arabidopsis histidine kinase CKI1 acts upstream of histidine phosphotransfer proteins to regulate female gametophyte development and vegetative growth. Plant Cell 22, 1232-1248. doi: 10.1105/tpc.108.065128

Denninger, P., Bleckmann, A., Lausser, A., Vogler, F., Ott, T., Ehrhardt, D. W., et al. (2014). Male-female communication triggers calcium signatures during fertilization in Arabidopsis. Nat. Commun. 5:4645.

El-Kasmi, F., Pacher, T., Strompen, G., Stierhof, Y. D., Müller, L. M., Koncz, C., et al. (2011). Arabidopsis SNARE protein SEC22 is essential for gametophyte development and maintenance of Golgi-stack integrity. Plant J. 66, 268-279. doi: 10.1111/j.1365-313x.2011.04487.x

Erbasol Serbes, I., Palovaara, J., and Gross-Hardt, R. (2019). Development and function of the flowering plant female gametophyte. Curr. Top. Dev. Biol. 131, 401-434. doi: 10.1016/bs.ctdb.2018.11.016

Erdmann, R. M., Hoffmann, A., Walter, H. K., Wagenknecht, H. A., Groß-Hardt, R., and Gehring, M. (2017). Molecular movement in the Arabidopsis thaliana female gametophyte. Plant Reprod. 30, 141-146. doi: 10.1007/s00497-0170304-3

Friedman, W. E. (1990). Double fertilization in Ephedra, a nonflowering seed plant: its bearing on the origin of Angiosperms. Science 247, 951-954. doi: 10.1126/science.247.4945.951

Friedman, W. E. (1998). The evolution of double fertilization and endosperm: an historical perspective. Sex. Plant Reprod. 11, 6-16. doi: 10.1007/s004970050114

Friedman, W. E. (2006). Embryological evidence for developmental lability during early angiosperm evolution. Nature 441, 337-340. doi: 10.1038/nature04690

Friedman, W. E. (2008). Hydatellaceae are water lilies with gymnospermous tendencies. Nature 453, 94-97. doi: 10.1038/nature06733

Friedman, W. E. (2015). Development and evolution of the female gametophyte and fertilization process in Welwitschia mirabilis (Welwitschiaceae). Am. J. Bot. 102, 312-324. doi: 10.3732/ajb.1400472

Friedman, W. E., Madrid, E. N., and Williams, J. H. (2008). Origin of the fittest and survival of the fittest: relating female gametophyte development to endosperm genetics. Int. J. Plant Sci. 169, 79-92. doi: 10.1086/523354

Friedman, W. E., and Ryerson, K. C. (2009). Reconstructing the ancestral female gametophyte of angiosperms: insights from Amborella and other ancient lineages of flowering plants. Am. J. Bot. 96, 129-143. doi: 10.3732/ajb.0800311

Friedman, W. E., and Williams, J. H. (2004). Developmental evolution of the sexual process in ancient flowering plant lineages. Plant Cell 16(Suppl.), S119-S132.

Garcia-Aguilar, M., Michaud, C., Leblanc, O., and Grimanelli, D. (2010). Inactivation of a DNA methylation pathway in maize reproductive organs results in apomixis-Like phenotypes. Plant Cell 22, 3249-3267. doi: 10.1105/ tpc.109.072181

Gonzalez, A., Sato, H., and Marazzi, B. (2019). Embryology in Helosis cayennensis (Balanophoraceae): structure of female flowers, fruit, endosperm and embryo. Plants 8:74. doi: 10.3390/plants8030074

Grossniklaus, U. (2017). Polyspermy produces tri-parental seeds in maize. Curr. Biol. 27, R1300-R1302.

Groß-Hardt, R., Kagi, C., Baumann, N., Moore, J. M., Baskar, R., Gagliano, W. B., et al. (2007). LACHESIS restricts gametic cell fate in the female gametophyte of Arabidopsis. PLoS Biol. 5:e47. doi: 10.1371/journal.pbio.0050047

Hamamura, Y., Nishimaki, M., Takeuchi, H., Geitmann, A., Kurihara, D., and Higashiyama, T. (2014). Live imaging of calcium spikes during double fertilization in Arabidopsis. Nat. Commun. 5:4722. 
Han, Y. Z., Huang, B. Q., Zee, S. Y., and Yuan, M. (2000). Symplastic communication between the central cell and the egg apparatus cells in the embryo sac of Torenia fournieri Lind. before and during fertilization. Planta 211, 158-162. doi: 10.1007/s004250000289

Hejátko, J., Pernisová, M., Eneva, T., Palme, K., and Brzobohat $\iota$, B. (2003). The putative sensor histidine kinase CKI1 is involved in female gametophyte development in Arabidopsis. Mol. Gen. Genomics 269, 443-453. doi: 10.1007/ s00438-003-0858-7

Ibarra, C. A., Feng, X., Schoft, V. K., Hsieh, T. F., Uzawa, R., Rodrigues, J. A., et al. (2012). Active DNA demethylation in plant companion cells reinforces transposon methylation in gametes. Science 337, 1360-1364. doi: 10.1126/ science. 1224839

Ingouff, M., Rademacher, S., Holec, S., Soljic, L., Xin, N., Readshaw, A., et al. (2010). Zygotic resetting of the HISTONE 3 variant repertoire participates in epigenetic reprogramming in Arabidopsis. Curr. Biol. 20, 2137-2143. doi: 10.1016/j.cub.2010.11.012

Kägi, C., Baumann, N., Nielsen, N., Stierhof, Y. D., and Groß-Hardt, R. (2010). The gametic central cell of Arabidopsis determines the lifespan of adjacent accessory cells. Proc. Natl. Acad. Sci. U.S.A. 107, 22350-22355. doi: 10.1073/ pnas. 1012795108

Kägi, C., and Groß-Hardt, R. (2007). How females become complex: cell differentiation in the gametophyte. Curr. Opin. Plant Biol. 10, 633-638. doi: 10.1016/j.pbi.2007.07.011

Kakimoto, T. (1996). CKI1, a histidine kinase homolog implicated in cytokinin signal transduction. Science 274, 982-985. doi: 10.1126/science.274.5289.982

Kawashima, T., and Berger, F. (2014). Epigenetic reprogramming in plant sexual reproduction. Nat. Rev. Genet. 15, 613-624. doi: 10.1038/nrg3685

Kirioukhova, O., Johnston, A. J., Kleen, D., Kagi, C., Baskar, R., Moore, J. M., et al. (2011). Female gametophytic cell specification and seed development require the function of the putative Arabidopsis INCENP ortholog WYRD. Development 138, 3409-3420. doi: 10.1242/dev.060384

Köhler, C., Wolff, P., and Spillane, C. (2012). Epigenetic mechanisms underlying genomic imprinting in plants. Annu. Rev. Plant Biol. 63, 331-352. doi: 10.1146/ annurev-arplant-042811-105514

Kong, J., Lau, S., and Jürgens, G. (2015). Twin plants from supernumerary egg cells in Arabidopsis. Curr. Biol. 25, 225-230. doi: 10.1016/j.cub.2014.11.021

Krohn, N. G., Lausser, A., Juraniæ, M., and Dresselhaus, T. (2012). Egg cell signaling by the secreted peptide ZmEAL1 controls antipodal cell fate. Dev. Cell 23, 219-225. doi: 10.1016/j.devcel.2012.05.018

Lafon-Placette, C., Johannessen, I. M., Hornslien, K. S., Ali, M. F., Bjerkan, K. N., Bramsiepe, J., et al. (2017). Endosperm-based hybridization barriers explain the pattern of gene flow between Arabidopsis lyrata and Arabidopsis arenosa in Central Europe. Proc. Natl. Acad. Sci. U.S.A. 114, E1027.

Li, H. J., Meng, J. G., and Yang, W. C. (2018). Multilayered signaling pathways for pollen tube growth and guidance. Plant Reprod. 31, 31-41. doi: 10.1007/ s00497-018-0324-7

Li, H. J., Zhu, S. S., Zhang, M. X., Wang, T., Liang, L., Xue, Y., et al. (2015). Arabidopsis CBP1 is a novel regulator of transcription initiation in central cellmediated pollen tube guidance. Plant Cell 27, 2880-2893. doi: 10.1105/tpc.15. 00370

Lituiev, D. S., Krohn, N. G., Müller, B., Jackson, D., Hellriegel, B., Dresselhaus, T., et al. (2013). Theoretical and experimental evidence indicates that there is no detectable auxin gradient in the angiosperm female gametophyte. Development 140, 4544-4553. doi: 10.1242/dev.098301

Liu, Z., Yuan, L., Song, X., Yu, X., and Sundaresan, V. (2017). AHP2, AHP3, and AHP5 act downstream of CKI1 in Arabidopsis female gametophyte development. J. Exp. Bot. 68, 3365-3373. doi: 10.1093/jxb/erx181

Lora, J., Yang, X., and Tucker, M. R. (2019). Establishing a framework for female germline initiation in the plant ovule. J. Exp. Bot. 70, 2937-2949. doi: 10.1093/ $\mathrm{jxb} / \mathrm{erz} 212$

Mao, Y., Gabel, A., Nakel, T., Viehöver, P., Baum, T., Tekleyohans, D. G., et al. (2020). Selective egg cell polyspermy bypasses the triploid block. eLife 9:e52976.

Martin, M. V., Fiol, D. F., Sundaresan, V., Zabaleta, E. J., and Pagnussat, G. C. (2013). oiwa, a female gametophytic mutant impaired in a mitochondrial manganese-superoxide dismutase, reveals crucial roles for reactive oxygen species during embryo sac development and fertilization in Arabidopsis. Plant Cell 25, 1573-1591. doi: 10.1105/tpc.113.109306
Márton, M. L., Cordts, S., Broadhvest, J., and Dresselhaus, T. (2005). Micropylar pollen tube guidance by egg apparatus 1 of maize. Science 307, 573-576. doi: $10.1126 /$ science. 1104954

Maruyama, D., Endo, T., and Nishikawa, S. (2010). BiP-mediated polar nuclei fusion is essential for the regulation of endosperm nuclei proliferation in Arabidopsis thaliana. Proc. Natl. Acad. Sci. U.S.A. 107, 1684-1689. doi: 10.1073/ pnas.0905795107

Maruyama, D., Yamamoto, M., Endo, T., and Nishikawa, S. (2014). Different sets of ER-resident J-proteins regulates distinct polar nuclear-membrane fusion events in Arabidopsis thaliana. Plant Cell Physiol. 55, 1937-1944. doi: 10.1093/pcp/ pcu120

Meng, J. G., Zhang, M. X., Yang, W. C., and Li, H. J. (2019). TICKET attracts pollen tubes and mediates reproductive isolation between relative species in Brassicaceae. Sci. China Life Sci. 62, 1413-1419. doi: 10.1007/s11427-019-98333

Mol, R., Matthys-Rochon, E., and Dumas, C. (1994). The kinetics of cytological events during double fertilization in Zea mays L. Plant J. 5, 197-206. doi: 10.1046/j.1365-313x.1994.05020197.x

Moll, C., von Lyncker, L., Zimmermann, S., Kägi, C., Baumann, N., Twell, D., et al. (2008). CLO/GFA1 and ATO are novel regulators of gametic cell fate in plants. Plant J. 56, 913-921. doi: 10.1111/j.1365-313x.2008.03650.x

Mori, T., Igawa, T., Tamiya, G., Miyagishima, S. Y., and Berger, F. (2014). Gamete attachment requires GEX2 for successful fertilization in Arabidopsis. Curr. Biol. 24, 170-175. doi: 10.1016/j.cub.2013.11.030

Pagnussat, G. C., Alandete-Saez, M., Bowman, J. L., and Sundaresan, V. (2009). Auxin-dependent patterning and gamete specification in the Arabidopsis female gametophyte. Science 324, 1684-1689. doi: 10.1126/science.1167324

Pagnussat, G. C., Yu, H. J., and Sundaresan, V. (2007). Cell-fate switch of synergid to egg cell in Arabidopsis eostre mutant embryo sacs arises from misexpression of the BEL1-Like homeodomain gene BLH1. Plant Cell 19, 3578-3592. doi: 10.1105/tpc.107.054890

Panoli, A., Martin, M. V., Alandete-Saez, M., Simon, M., Neff, C., Swarup, R., et al. (2015). Auxin import and local auxin biosynthesis are required for mitotic divisions, cell expansion and cell specification during female gametophyte development in Arabidopsis thaliana. PLoS One 10:e0126164. doi: 10.1371/ journal.pone. 0126164

Park, K., Kim, M. Y., Vickers, M., Park, J. S., Hyun, Y., Okamoto, T., et al. (2016). DNA demethylation is initiated in the central cells of Arabidopsis and rice. Proc. Natl. Acad. Sci. U.S.A. 113, 15138-15143. doi: 10.1073/pnas.1619047114

Pillot, M., Baroux, C., Vazquez, M. A., Autran, D., Leblanc, O., Vielle-Calzada, J. P., et al. (2010). Embryo and endosperm inherit distinct chromatin and transcriptional states from the female gametes in Arabidopsis. Plant Cell 22, 307-320. doi: 10.1105/tpc.109.071647

Pischke, M. S., Jones, L. G., Otsuga, D., Fernandez, D. E., Drews, G. N., and Sussman, M. R. (2002). An Arabidopsis histidine kinase is essential for megagametogenesis. Proc. Natl. Acad. Sci. U.S.A. 99, 15800-15805. doi: 10. 1073/pnas.232580499

Portereiko, M. F., Lloyd, A., Steffen, J. G., Punwani, J. A., Otsuga, D., and Drews, G. N. (2006). AGL80 is required for central cell and endosperm development in Arabidopsis. Plant Cell 18, 1862-1872. doi: 10.1105/tpc.106.040824

Povilus, R. A., Losada, J. M., and Friedman, W. E. (2015). Floral biology and ovule and seed ontogeny of Nymphaea thermarum, a water lily at the brink of extinction with potential as a model system for basal angiosperms. Ann. Bot. 115, 211-226. doi: 10.1093/aob/mcu235

Rabiger, D. S., and Drews, G. N. (2013). MYB64 and MYB119 are required for cellularization and differentiation during female gametogenesis in Arabidopsis thaliana. PLoS Genet. 9:e1003783. doi: 10.1371/journal.pgen.1003783

Raissig, M. T., Bemer, M., Baroux, C., and Grossniklaus, U. (2013). Genomic imprinting in the Arabidopsis embryo is partly regulated by PRC2. PLoS Genet. 9:e1003862. doi: 10.1371/journal.pgen.1003862

Schmid, M. W., Schmidt, A., and Grossniklaus, U. (2015). The female gametophyte: an emerging model for cell type-specific systems biology in plant development. Front. Plant Sci. 6:907. doi: 10.3389/fpls.2015.00907

Schmidt, A., Wöhrmann, H. J., Raissig, M. T., Arand, J., Gheyselinck, J., Gagliardini, V., et al. (2013). The Polycomb group protein MEDEA and the DNA methyltransferase MET1 interact to repress autonomous endosperm development in Arabidopsis. Plant J. 73, 776-787. doi: 10.1111/tpj.12070 
Scott, R. J., Armstrong, S. J., Doughty, J., and Spielman, M. (2008). Double fertilization in Arabidopsis thaliana involves a polyspermy block on the egg but not the central cell. Mol. Plant 1, 611-619. doi: 10.1093/mp/ssn016

Shi, D. Q., and Yang, W. C. (2011). Ovule development in Arabidopsis: progress and challenge. Curr. Opin. Plant Biol. 14, 74-80. doi: 10.1016/j.pbi.2010.09.001

Shimizu, K. K., Ito, T., Ishiguro, S., and Okada, K. (2008). MAA3 (MAGATAMA3) helicase gene is required for female gametophyte development and pollen tube guidance in Arabidopsis thaliana. Plant Cell Physiol. 9, 1478-1483. doi: 10.1093/ $\mathrm{pcp} / \mathrm{pcn} 130$

Soma, S. (1997). "Development of the female gametophyte and the embryogeny of Ginkgo biloba," in Ginkgo Biloba: A Global Treasure, eds T. Hori, R. W. Ridge, W. Tulecke, P. Del Tredici, J. Trémouillaux-Guiller, and H. Tobe (Tokyo: Springer).

Steffen, J. G., Kang, I. H., Portereiko, M. F., Lloyd, A., and Drews, G. N. (2008). AGL61 interacts with AGL80 and is required for central cell development in Arabidopsis. Plant Physiol. 148, 259-268. doi: 10.1104/pp.108.119404

Sundaresan, V., and Alandete-Saez, M. (2010). Pattern formation in miniature: the female gametophyte of flowering plants. Development 137, 179-189. doi: 10.1242/dev.030346

Susaki, D., Suzuki, T., Maruyama, D., Ueda, M., Higashiyama, T., and Kurihara, D. (2020). Dynamics of the cell fate specifications during female gametophyte development in Arabidopsis. bioRxiv [Preprint]. doi: 10.1101/2020.04.07. 023028

Susaki, D., Takeuchi, H., Tsutsui, H., Kurihara, D., and Higashiyama, T. (2015). Live Imaging and Laser disruption reveal the dynamics and cell-cell communication during Torenia fournieri female gametophyte development. Plant Cell Physiol. 56, 1031-1041. doi: 10.1093/pcp/pcv031

Tekleyohans, D. G., Nakel, T., and Groß-Hardt, R. (2017). Patterning the female gametophyte of flowering plants. Plant Physiol. 173, 122-129. doi: 10.1104/pp. 16.01472

Völz, R., von Lyncker, L., Baumann, N., Dresselhaus, T., Sprunck, S., and Groß-Hardt, R. (2012). LACHESIS-dependent egg-cell signaling regulates the development of female gametophytic cells. Development 139, 498-502. doi: 10.1242/dev.075234

Wang, D., Lu, Y., Zhang, M., Lu, Z., Luo, K., Cheng, F., et al. (2014). Structure and function of the neck cell during fertilization in Ginkgo biloba L. Trees 28, 995-1005. doi: 10.1007/s00468-014-1013-2

Weijers, D. (2016). A female identity switch helps keep only one egg in the basket. Dev. Cell 37, 5-6. doi: 10.1016/j.devcel.2016.03.011
Wu, J. J., Peng, X. B., Li, W. W., He, R., Xin, H. P., and Sun, M. X. (2012). Mitochondrial GCD1 dysfunction reveals reciprocal cell-to-cell signaling during the maturation of Arabidopsis female gametes. Dev. Cell 23, 1043-1058. doi: 10.1016/j.devcel.2012.09.011

Yadegari, R., and Drews, G. N. (2004). Female gametophyte development. Plant Cell 16(Suppl. 1):S133.

Yamada, H., Suzuki, T., Terada, K., Takei, K., Ishikawa, K., Miwa, K., et al. (2001). The Arabidopsis AHK4 histidine kinase is a cytokinin-binding receptor that transduces cytokinin signals across the membrane. Plant Cell Physiol. 42, 1017-1023. doi: 10.1093/pcp/pce127

Yang, W. C., Shi, D. Q., and Chen, Y. H. (2010). Female gametophyte development in flowering plants. Annu. Rev. Plant Biol. 61, 89-108. doi: 10.1146/annurevarplant-042809-112203

Yuan, L., Liu, Z., Song, X., Jernstedt, J., and Sundaresan, V. (2018). The gymnosperm ortholog of the angiosperm central cell-specification gene CKI1 provides an essential clue to endosperm origin. New Phytol. 218, 1685-1696. doi: 10.1111/nph.15115

Yuan, L., Liu, Z., Song, X., Johnson, C., Yu, X., and Sundaresan, V. (2016). The CKI1 histidine kinase specifies the female gametic precursor of the endosperm. Dev. Cell 37, 34-46. doi: 10.1016/j.devcel.2016.03.009

Zhang, M. X., Zhu, S. S., Xu, Y. C., Guo, Y. L., Yang, W. C., and Li, H. J. (2020). Transcriptional repression specifies the central cell for double fertilization. Proc. Natl. Acad. Sci. U.S.A. 117, 6231-6236. doi: 10.1073/pnas.19094 65117

Zini, L. M., Galati, B. G., Ferrucci, M. S., Zarlavsky, G., and Rosenfeldt, S. (2016). Ultrastructural study of the female gametophyte and the epistase in Cabombaceae and Nymphaeaceae. Flora 220, 25-36. doi: 10.1016/j.flora.2016. 02.006

Conflict of Interest: The authors declare that the research was conducted in the absence of any commercial or financial relationships that could be construed as a potential conflict of interest.

Copyright (c) $2020 \mathrm{Li}$ and Yang. This is an open-access article distributed under the terms of the Creative Commons Attribution License (CC BY). The use, distribution or reproduction in other forums is permitted, provided the original author(s) and the copyright owner(s) are credited and that the original publication in this journal is cited, in accordance with accepted academic practice. No use, distribution or reproduction is permitted which does not comply with these terms. 